\title{
Trombone Synthesis by Model and Measurement
}

\author{
Tamara Smyth and Frederick S. Scott \\ School of Computing Science, Simon Fraser University, Surrey, BC, Canada V3T 0A3 \\ Correspondence should be addressed to Tamara Smyth, tamaras@cs.sfu.ca
}

Received 29 August 2010; Revised 15 December 2010; Accepted 24 January 2011

Academic Editor: Vesa Valimaki

Copyright (C) 2011 T. Smyth and F. S. Scott. This is an open access article distributed under the Creative Commons Attribution License, which permits unrestricted use, distribution, and reproduction in any medium, provided the original work is properly cited.

\begin{abstract}
A physics-based synthesis model of a trombone is developed using filter elements that are both theoretically-based and estimated from measurement. The model consists of two trombone instrument transfer functions: one at the position of the mouthpiece enabling coupling to a lip-valve model and one at the outside of the bell for sound production. The focus of this work is on extending a previously presented measurement technique used to obtain acoustic characterizations of waveguide elements for cylindrical and conical elements, with further development allowing for the estimation of the flared trombone bell reflection and transmission functions for which no one-parameter traveling wave solution exists. A one-dimensional bell model is developed providing an approximate theoretical expectation to which estimation results may be compared. Dynamic trombone model elements, such as those dependent on the bore length, are theoretically and parametrically modeled. As a result, the trombone model focuses on accuracy, interactivity, and efficiency, making it suitable for a number of real-time computer music applications.
\end{abstract}

\section{Introduction}

Instrument synthesis involving real-time interactive sound often faces trade-offs between accuracy and computational efficiency to provide both parametric control and quality sound production. It is often the case that a more playable model, one that is more responsive to human gestural input, is a better sounding virtual musical instrument than one that prioritizes acoustic precision. That is, the more the sound production can be effectively controlled in the hands of a musician employing phrasing, nuances, and other musical subtleties, the more the perceived sound quality will approach that of an actual acoustic instrument. Nevertheless, producing a model that is as acoustically accurate as resources will allow requires knowledge of the instrument's acoustic characteristics, properties that may be effectively obtained by measurement.

Acoustic accuracy becomes increasingly important if the focus of the model's application is less on interactive sound production and more on model validation, inverse modeling, and parameter estimation. For example, if the goal is to extract physical parameter values from an instrument during performance, the model's produced sound when played with proper parameter values will likely require a higher degree of actual (over perceived) similarity to the instrument being modeled. In this application, which is gaining increasing attention in the physical modeling community [1-8], the virtual model must often also account for the frequency characteristics of any variables involved in the acquisition of data, such as instrument radiation, mic placement, or inclusion of any measurement device/apparatus that may also alter the acoustic behaviour of the instrument being modeled.

In this work, a physics-based synthesis model of a trombone is presented, suitable for applications mentioned above. That is, the aim is for high-quality real-time sound production, with highly intuitive and interactive control parameters, yet with a sufficiently accurate acoustic model of the trombone instrument that its inverse transfer function may be applied during real-time performance. Deconvolving the effects of the bore and bell in the instrument's produced sound allows for the estimation of the dynamic effects of the lip-valve signal, a signal related to the valve's volume velocity holding the primary sound control information for most wind instruments such as blowing pressure, embouchure, and more advanced playing techniques. The instrument's 
produced sound may be seen as the coupling of the pressure input from the lips (the product of the volume velocity and the bore opening's characteristic impedance) with the instrument bore and bell-a convolution of the lip-valve signal and the trombone impulse response. With this view, isolation of the lip-valve signal is a matter of obtaining and deconvolving the instrument's transfer function, as well as any measurement variables, from the instrument's produced sound.

The focus herein, therefore, is on obtaining a parametric model of the trombone's transfer function in two positions: one tapped at the position of the mouth and the other outside the bell. The former may be coupled to a lip-valve model, providing feedback of bore resonances and the pressure difference across the lip valve (required for dynamic models in which the bore pressure influences the behaviour of the vibrating lips [9]), while the latter may be convolved with the lip-valve signal to provide the instrument's produced sound. In both cases, a low-latency convolution operation must be used, with the delay being less than one round-trip from mouthpiece to bell and back, in order to yield the accuracy of its waveguide counterpart when coupling the lip valve to the bore $[10,11]$. Both transfer functions are expressed parametrically, allowing for any necessary change in their frequency response during performance.

To this end, the instrument transfer functions are generalized to be valid for three physically assembled instrument structures having incremental resemblance to the final complete trombone: (1) a two-meter long cylindrical tube with a closed end (I1 in Section 3), (2) the same cylindrical tube with an open end (I2 in Section 3), and (3) the same cylindrical tube with a trombone bell (I4 in Section 3). The mouthpiece is omitted because its structure is not constant during performance (as is the assumption made of the trombone bore and bell). The vibrating lips create a timevarying volume and opening in the mouthpiece, making it difficult to characterize using the methods described here.

The model employs a measurement and a processing technique from previous work [12], whereby waveguide elements are estimated from several measurements of the system's impulse response, with the system having incrementally varying boundary conditions to allow for the isolation and estimation of filter transfer functions. The method as described in [12] is explored using simple cylindrical and conical tube structures, as these are well described theoretically and provide a basis for validating measured and estimated data. The method is extended and applied here to measure instrument structures that are more difficult to account for theoretically, and which are not expected to change during performance, such as the reflection and transmission of the trombone's bell, with comparison to classic modeling techniques showing consistency in the results.

\section{Trombone Instrument Model}

It is well known that wave propagation in wind instrument bores may be modeled in one dimension using the waveguide structure shown in Figure 1, with a bidirectional delay line of

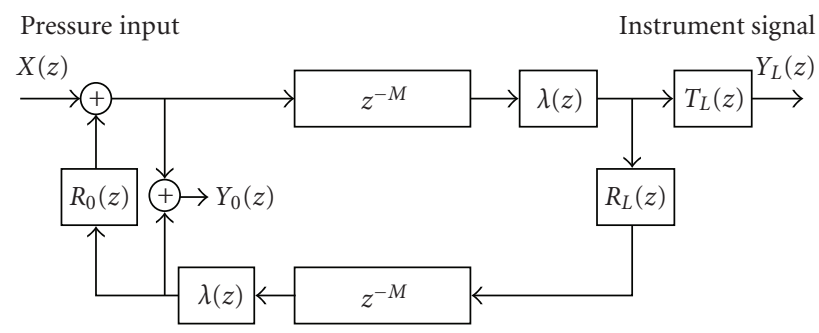

Figure 1: Waveguide model of a cylindrical tube with commuted propagation loss filters $\lambda(z)$ and open-end terminating reflection and transmission filters $R_{L}(z)$ and $T_{L}(z)$, respectively, and a reflection filter $R_{0}(z)$ at the (effectively) closed-end termination corresponding to the position of the mouthpiece. Two instrument transfer functions (1) and (2) are developed for observation points yielding $Y_{0}(z)$ and $Y_{L}(z)$, corresponding to the bore base and the instrument output, respectively, in response to input pressure $X(z)$.

length $M$ samples accounting for the acoustic propagation delay in the cylindrical and/or conical tube section of a given length and filter elements $\lambda(z), R_{0}(z), R_{L}(z)$, and $T_{L}(z)$, accounting for the propagation loss, reflection at the mouthpiece, and open-end reflection and transmission occurring at the position of the bell, respectively, all of which may contain delays, poles, or "long-memory" information on the acoustics of the noncylindrical/nonconical bore section $[13,14]$.

The bidirectional delay line is suitable for modeling the left and right travelling waves in both cylindrical and conical tube structures, for which exact analytic solutions are known and can be derived by solving the wave equation. When tube contours depart from these simple shapes, however, the model must account for the continuous reflection and transmission that is distributed along the length of the changing cross-sectional area. For the flared opening of many brass instruments, such as the trombone bell, it has been shown that no one-parameter traveling wave solution exists [15-17]. Nevertheless, computationally efficient approximations may be made using one-dimensional models, such as those using Webster's equation for adapted coordinates $[15,17]$, or a piecewise connection of several cylindrical segments (discontinuous approximation [13]) and/or conical segments (continuous approximation $[18,19]$ ), or "constant curved" ( $\mathcal{C}^{1}$-regular approximation [20]) corresponding to the instrument's profile. As wave propagation within horns involves an admixture of higher-order and/or evanescent modes, a one-dimensional model cannot capture the complete behaviour. Another approach therefore, shown in Figure 1, is to separate a horn into its cylindrical/conical and flared sections, with lumped filters accounting for the reflection and transmission of the flared bell. It has been repeatedly observed that these filters tend to be minimumphase $[12,21]$, and thus it is hypothesized that these filters possess this property, making them, by definition, well posed in terms of causality and stability.

In the case of the lumped model, reflection and transmission filter properties of the bell, which contribute significantly to the instrument model's characteristic resonances, 
may be obtained theoretically or by acoustic measurement $[12,22-26]$. The latter is particularly beneficial if the section need not be parametric because it is not expected to change during instrument performance. Because a measured frequency response is not limited by theoretical approximations for propagation losses or those occurring at the open-end boundary, it is expected to provide greater accuracy for waveguide elements such as the bore and bell-provided the method for obtaining their frequency response is valid.

As is mentioned in Section 1, three structures bearing incremental resemblance to the complete trombone instrument are constructed, all of which may be modeled using the waveguide structure shown in Figure 1, with the characteristic waveguide elements being changed according to tube/bore width and length and the conditions at the boundaries (observation points at the position of the mouthpiece and outside the bell). The incremental measurements allow for the isolation and estimation of each waveguide element, including variables in the measurement setup, such as the speaker transmission and reflection functions.

As mentioned above, depending on the method being used for modeling the lip valve, it is useful to tap the signal flow diagram in Figure 1 at two different positions: at the bore base, yielding pressure $Y_{0}(z)$, and at the instrument output, yielding transmitted pressure $Y_{L}(z)$, both in response to input pressure $X(z)$ at the bore base (position of the mouthpiece). This yields two separate instrument transfer functions for both coupling to the lip valve and producing the instrument's sound output. Ignoring the time-varying component in the mouthpiece, computing the global transfer function $H=Y_{0} / X$ at the bore base (position of the mouthpiece) in the $z$-domain, yields

$$
H(z)=\frac{Y_{0}(z)}{X(z)}=\frac{1+\lambda^{2}(z) R_{L}(z) z^{-2 M}}{1-\lambda^{2}(z) R_{L}(z) R_{0}(z) z^{-2 M}},
$$

where $\lambda(z)$ is the propagation loss and $R_{0}(z)$ and $R_{L}(z)$ are the reflection functions describing the boundaries at the position of mouthpiece and bell, respectively. Similarly, computing the global transfer function $G=Y_{L} / X$ at the instrument output in the $z$-domain yields

$$
G(z)=\frac{Y_{L}(z)}{X(z)}=\frac{T_{L}(z) \lambda(z) z^{-M}}{1-\lambda^{2}(z) R_{L}(z) R_{0}(z) z^{-2 M}} .
$$

For all $z$, such that $|z|<1,\left|\lambda^{2}(z) R_{L}(z) R_{0}(z)\right|<1$, a series expansion of (1) and (2),

$$
\begin{aligned}
Y_{0}(z)= & X(z)\left(1+\lambda^{2}(z) R_{L}(z) z^{-2 M}\right) \\
\times & \quad\left[1+R_{0}(z) R_{L}(z) \lambda^{2}(z) z^{-2 M}\right. \\
& \left.\quad+R_{0}^{2}(z) R_{L}^{2}(z) \lambda^{4}(z) z^{-4 M}+\cdots\right], \\
Y_{L}(z)= & X(z) T_{L}(z) \lambda(z) z^{-M} \\
\times & {\left[1+R_{0}(z) R_{L}(z) \lambda^{2}(z) z^{-2 M}\right.} \\
& \left.\quad+R_{0}^{2}(z) R_{L}^{2}(z) \lambda^{4}(z) z^{-4 M}+\cdots\right],
\end{aligned}
$$

makes a standard train-wave decomposition appear.

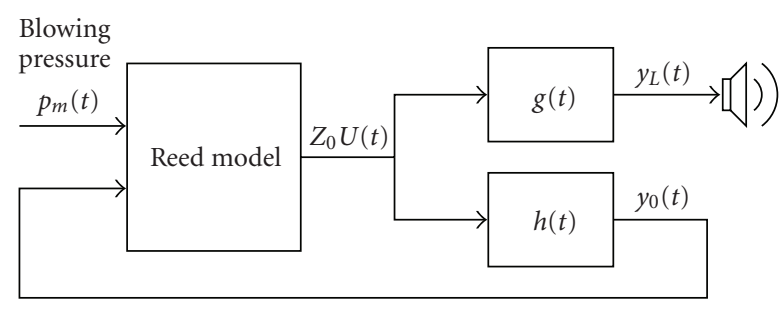

FIGURE 2: A convolutional synthesis approach to the signal flow diagram shown in Figure 1, with signals $h(t)$ and $g(t)$ being the impulse responses of the instrument tapped at the positions $y_{0}(t)$ and $y_{L}(t)$, the inverse transforms of (1) and (2), respectively. The input pressure is the product of the characteristic (wave) impedance $Z_{0}$ at the mouthpiece and the volume flow $U(t)$, a signal generated by a reed model in response to a blowing pressure $p_{m}(t)$.

Expressing the instrument model in this way conveniently allows the outputs shown in Figure 1 to be simulated as the convolution of the input and the instrument transfer functions at these two positions, as shown in Figure 2, where $h(t)$ and $g(t)$ are the inverse Fourier transforms of the frequency responses corresponding to (1) and (2), respectively.

\section{Measurement Setup}

Filters for the equivalent digital waveguide and convolutional synthesis models (Figures 1 and 2) described by instrument transfer functions (1) and (2) are estimated from measurement using a technique introduced in $[12,27]$ and later applied in [28]. The technique uses a method similar to [29, 30] for extracting reflection functions using train-wave-like decomposition. In $[29,30]$, reflection functions are obtained for a complete instrument (combined bore and bell) by measuring a B-flat trumpet and then made parametric by altering the delay time between arrivals to simulate a change in pitch-simulating a slide trumpet. In the method here, however, individual waveguide elements corresponding to those in Figure 1 are estimated from arrivals in several instances of the system's measured impulse response having incrementally varying terminating/boundary conditions. Since the bell may be removed from the trombone, its reflection and transmission functions may be estimated using the measurement system described herein.

3.1. Measurement System Specifications. A test signal, a logarithmic swept sinusoid of sufficient length (20 seconds) to ensure a sufficiently large signal-to-noise ratio (SNR) [31], drives a speaker (CUI Inc. CMS020KLX) that seals one end of a two-meter-long cylindrical acoustic tube. Audio (24 bit/44.1 kHz) input/output is done using a MOTU Traveler mk3.

A microphone (JL-061C) is press fit into a hole in the tube wall, as close to the speaker as possible and flush with the tube interior wall (see Figure 3), to capture the signal $y_{0}(t)$ - the pressure consisting of the sum of right and left travelling waves - in response to the test signal transmitted from the speaker. A second microphone (same model) is 

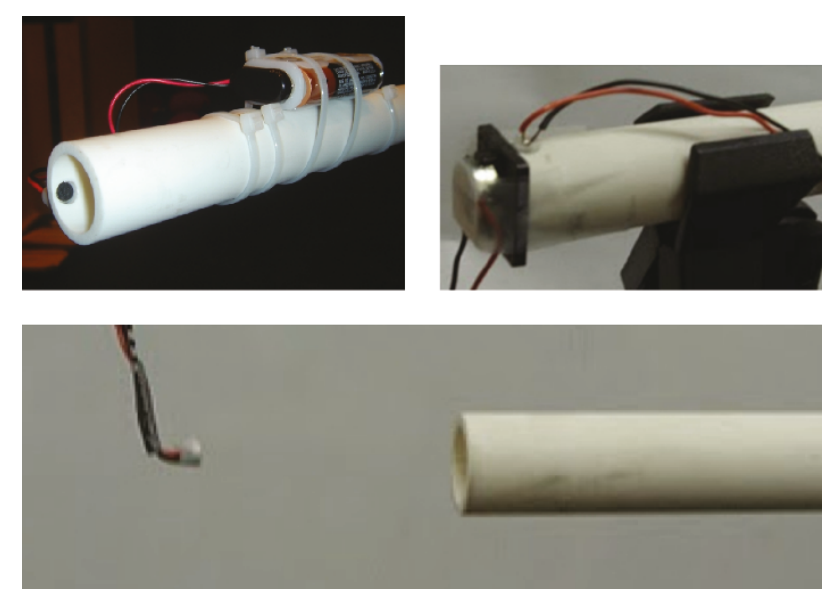

Figure 3: A speaker seals one end of a two-meter long cylindrical tube, with a colocated microphone press fit into the tube wall, flush with the tube's interior wall. A second microphone is placed $7 \mathrm{~cm}$ outside the instrument/tube's open end, on the tube/bore axis. This setup may be modeled using the signal flow diagram in Figure 4.

placed $7 \mathrm{~cm}$ outside of instrument's open end, on axis with the tube, to capture the signal $y_{L}(t)$. The peak level for $y_{0}(t)$ measured approximately $90 \mathrm{~dB}$ above the noise floor standard deviation, whereas the SNR for $y_{L}(t)$ was approximately $70 \mathrm{~dB}$, both for a 20 -second-long test signal.

As described in [31], the recorded signal is linearly deconvolved to separate nonlinear harmonic distortion (caused by the speaker) from the desired linear impulse response-the response of the system had it been excited with a pulse only one sample long (an ideal condition that would otherwise be physically unrealistic in band-limited systems). Experiments showed that by using this technique, measurements taken with varying levels of speaker distortion (including no distortion at all) have no visible effects on the results presented here.

The measurement setup is applied to the four incrementally constructed "instruments" I1, I2, I3, and I4, which may be modeled following Figure 4 and/or Figure 11, allowing for the confirmation of measurement consistency and the isolation and estimation of waveguide model elements that may be validated through comparison with theoretical expectation. All instruments consist of a two-meter-long cylindrical tube (PVC pipe) with a diameter of $2 \mathrm{~cm}$ and are terminated at one end with a speaker, with the opposite end having the following terminations:

I1: closed, producing a "perfect" reflection,

I2: open, producing a reflection and transmission for open cylinders,

I3: shorter cylinder affixed, for calculating cylindrical reflection and transmission in the presence of a 2port scattering junction between cylindrical sections,

I4: trombone bell affixed, producing a reflection and transmission having the bell's acoustic characteristics.

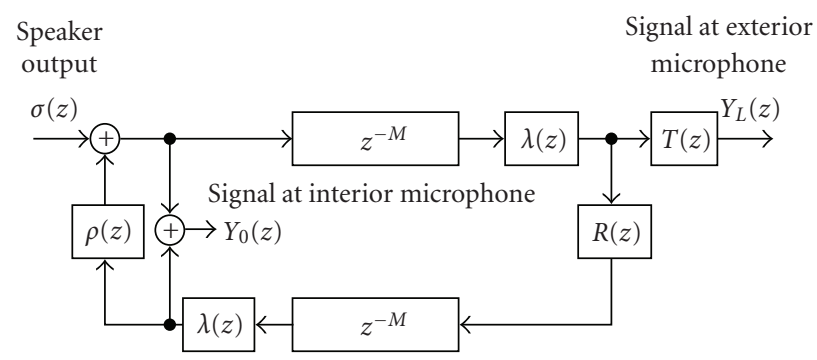

Figure 4: Waveguide model of a cylindrical tube adapted from Figure 1, with microphone capturing signal response $Y_{0}(z)$ at the bore base, and a colocated speaker having a transmission function of $\sigma(z)$ and a reflection function of $\rho(z)$. At the opposite end, there are three possible terminating conditions relating to instruments I1, I2, and I4: (1) I1: $R(\mathrm{z})=1$ and $T(z)=0$ for a perfectly closed tube, (2) I2: $R(z)=R_{L}(z)$ and amplitude complementary $T(z)=$ $T_{L}(z)=1+R_{L}(z)$ for an open cylinder, and (3) I4: $R(z)=R_{b}(z)$ and $T(z)=T_{b}(z)$ for the appended trombone bell. Instrument $\mathrm{I} 3$ is described by the diagram in Figure 11.

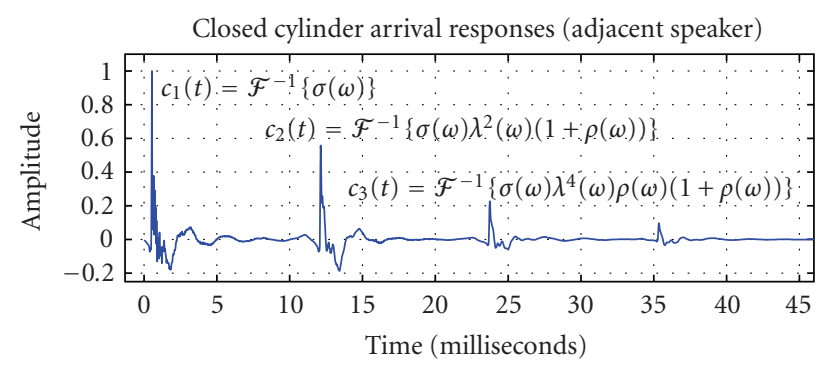

FIGURE 5: Arrival responses for closed cylinder, showing evenly spaced pulses as inverse transforms of combinations of transfer functions for the speaker $\sigma(\omega)$, propagation losses $\lambda(\omega)$, and speaker reflection $\rho(\omega)$.

The tube must be long enough to ensure that each arrival in the measured impulse responses decays before the onset of the next. As shown in Figures 5, 7, and 9, the two-meter tube is sufficiently long to satisfy these criteria, allowing arrival echoes to be windowed for extraction from the measured impulse response with negligible loss of information. A rectangle window with a taper at the tail provided by a raised cosine further ensures the complete decay of each echo.

The measurement technique applied to instruments I1 and I2 is described in this section, summarizing the measurement technique in [12] (but using current measurements), as well as providing a foundation and validation, as these structures are well described theoretically. Beginning each measurement session with measurements of I1 and I2 may be viewed as a calibration step to ensure consistency of measurements. Responses of I1 allow for the estimation of system variables, the speaker transmission $\hat{\rho}(\omega)$, estimated reflection off the speaker $\hat{\rho}(\omega)$, and the estimated propagation losses $\hat{\lambda}(\omega)$-all of which are necessary to estimate elements from subsequent measurements. Measurement of I2 (along with elements estimated from I1) allows for the estimation of the open-end reflection $R_{L}$ and transmission $T_{L}$. Both 


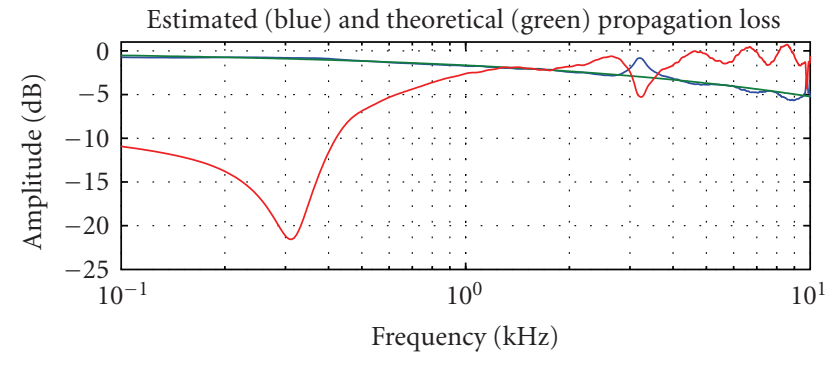

FIGURE 6: Theoretically modeled propagation loss (green smooth curve) [21] and estimated propagation loss $\hat{\lambda}(\omega)$ (blue) show a close fit, in spite of a far-from-ideal speaker reflection response $\rho(\omega)$ (red high-pass curve).

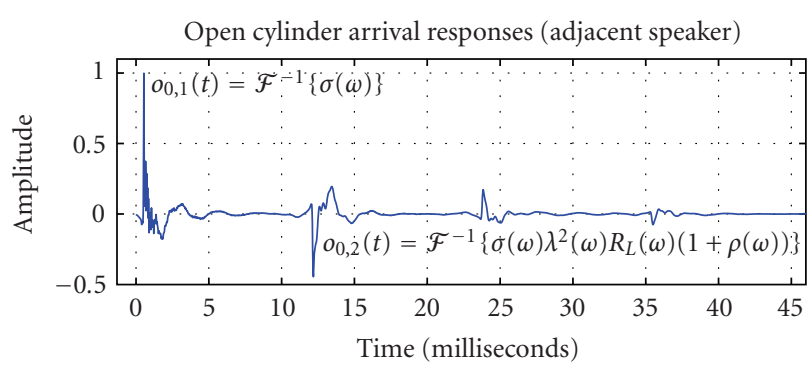

(a)

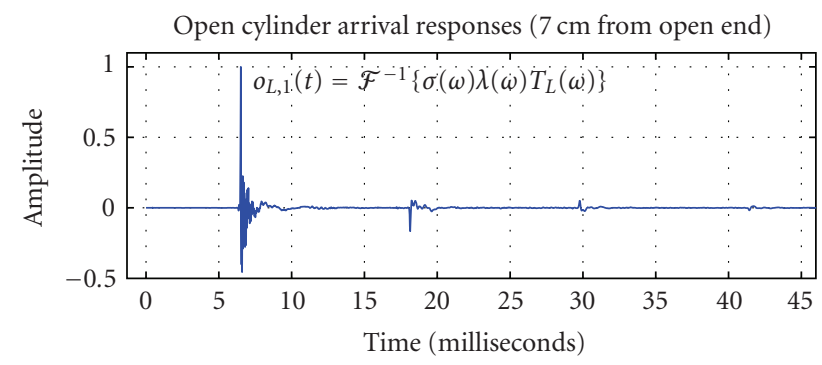

(b)

FIGURE 7: Arrival responses of the open cylinder at speaker (a) and open end (b), showing evenly spaced pulses as inverse transforms of combinations of transfer functions seen in Figure 5, with the addition of the open-end reflection $R_{L}(\omega)$ (a) and transmission $T_{L}(\omega)$ (b). Notice the alternating polarity (a) as compared to Figure 7, due to the negative reflection of an open end.

propagation losses $\lambda(\omega)$, reflection $R_{L}$, and transmission $T_{L}$ are compared with theory to ensure measurement accuracy.

Measurement of I4, formed by appending a trombone bell to the end of the two-meter tube, allows for the estimation of the trombone bell reflection and transmission functions using either the method used for estimating the open-end reflection for I2 or an improved method that accounts for the junction introduced by appending an acoustic object with a different cross-section than the measurement tube, as described in Section 4. Instrument I3, also described in Section 4, is an intermediary step used to validate the method of accounting for the junction by comparing the open cylinder reflection estimated from this measurement to that estimated from measurement of I2.
3.2. Estimating Speaker Transmission/Reflection and Propagation Losses from I1. In instrument I1, the two-meter-long tube is closed at both ends, with the speaker at one end and a rigid termination (a piece of plastic) producing a perfect reflection at the other. As seen in Figure 5, the measured response to the test signal at the inside microphone yields a sequence of uniformly spaced echoes or arrivals, separated by the time it takes the sound to travel the length of the tube and back, each one decaying almost completely before the onset of the next. The first three arrivals, each having ample SNR, are sufficient for estimating the speaker output $\sigma(\omega)$, the speaker reflection $\rho(\omega)$, and the propagation loss $\lambda(\omega)$ transfer functions.

Assuming both a microphone magnitude response that is flat in the band of interest and no prior circulating energy in the tube, the first arrival is simply the speaker output, the speaker transfer function, given by

$$
C_{1}(\omega)=\sigma(\omega)
$$

Assuming sufficient decay of $c_{1}(t)$ before the onset of $c_{2}(t)$, as observed in Figure 5, the second arrival is the sum of the incoming reflection from the closed end, $C_{2}^{-}(\omega)=$ $\sigma(\omega) \lambda^{2}(\omega)$, and the simultaneous reflection from the speaker, $C_{2}^{+}(\omega)=\sigma(\omega) \lambda^{2}(\omega) \rho(\omega)$, and is given by

$$
C_{2}(\omega)=C_{2}^{+}(\omega)+C_{2}^{-}(\omega)=\sigma(\omega) \lambda^{2}(\omega)(1+\rho(\omega)) .
$$

Like the second arrival, each subsequent arrival consists of the previous arrival with round-trip wall losses $\lambda^{2}(\omega)$ and a speaker reflection $\rho(\omega)$, yielding a third arrival given by

$$
C_{3}(\omega)=\sigma(\omega) \lambda^{4}(\omega) \rho(\omega)(1+\rho(\omega))
$$

An intermediate variable,

$$
\zeta(\omega)=\frac{C_{1}(\omega) C_{3}(\omega)}{\left(C_{2}(\omega)\right)^{2}}=\frac{\rho(\omega)}{1+\rho(\omega)},
$$

is then defined to yield estimates for the speaker reflection transfer function

$$
\hat{\rho}(\omega)=\frac{\zeta(\omega)}{1-\zeta(\omega)}
$$

and finally the round-trip propagation loss

$$
\hat{\lambda}^{2}(\omega)=\frac{C_{3}(\omega)}{\hat{\rho}(\omega) C_{2}(\omega)} .
$$

As shown in Figure 6, one-way estimated losses obtained using the square root of (9) show a very good fit to those modeled from theory as described in $[21,32]$.

3.3. Estimating Cylindrical Open-End Reflection and Transmission from I2. Taking another measurement after removing the rigid termination and leaving the tube open yields the sequence of arrivals seen in Figure 7, with the top showing the arrivals $o_{0, n}(t)$ at the mic adjacent to the speaker (corresponding to $y_{0}(t)$ ) and the bottom showing the arrivals $o_{L, n}(t)$ at the mic placed outside the tube, $7 \mathrm{~cm}$ 
from its open end (corresponding to $y_{L}(t)$ ). Using these measurements along with those made of the closed tube, allows for the estimation of the open-end reflection and transmission functions, $R_{L}(\omega)$ and $T_{L}(\omega)$, respectively.

The first arrival at the speaker location $O_{0,1}(\omega)$, like $C_{1}(\omega)$, is the speaker transfer function, and the comparison of the two is a useful way of ensuring consistency from one measurement to the next. The second arrival $\mathrm{O}_{0,2}(\omega)$ contains the same elements as $C_{2}(\omega)$ but with the additional contribution of the open-end reflection, $R_{L}(\omega)$ :

$$
O_{0,2}(\omega)=\sigma(\omega) \lambda^{2}(\omega) R_{L}(\omega)(1+\rho(\omega))
$$

Third and subsequent arrivals at this location follow in the same fashion, consisting of the previous arrival with additional accumulated round-trip losses as it propagates from the microphone to the open end and back again.

The open-end reflection may be estimated by taking the ratio of the second arrival spectra from the open and closed tubes:

$$
\hat{R}_{L}(\omega)=\frac{O_{0,2}(\omega)}{C_{2}(\omega)}=\frac{\sigma(\omega) \lambda^{2}(\omega) R_{L}(\omega)(1+\rho(\omega))}{\sigma(\omega) \lambda^{2}(\omega)(1+\rho(\omega))} .
$$

As shown in Figure 8(a), the estimate in (11) very closely matches the theoretical expectation described in [12].

The first arrival at the mic placed outside the tube, $7 \mathrm{~cm}$ from the open end is given by

$$
O_{L, 1}(\omega)=\sigma(\omega) \lambda(\omega) T_{L}(\omega)
$$

Dividing this first arrival spectrum by the product of the speaker transmission (4) and wall losses estimated using the square root of (9) yields the estimated transmission from an open cylinder

$$
\widehat{T}_{L}(\omega)=\frac{O_{L, 1}(\omega)}{C_{1}(\omega) \hat{\lambda}(\omega)} .
$$

As seen in Figure 8(c), the estimated transmission (13) shows a very close match to the amplitude complement of the theoretical reflection (the expected relationship between reflection and transmission for a cylindrical opening), validating the measurement taken from outside the tube's open end.

This section set up the measurement technique and showed impulse response measurements made from instruments I1 and I2, consisting of evenly spaced arrivals, from which the speaker transmission $\sigma(\omega)$ and reflection $\rho(\omega)$, cylindrical propagation loss $\lambda(\omega)$, open-end reflection $R_{L}(\omega)$, and $T_{L}(\omega)$, corresponding to waveguide elements in Figure 4, could be estimated. The method is validated by comparing propagation loss $\lambda(\omega)$ to theory described in [21] and the reflection/transmission to theory described in $[12,33]$. Though comparison is made using magnitudes of the frequency response, it should be noted that phase is also important. It has been found that these elements tend to be minimum phase, or approximately so, so that by definition they are stable and causal, and a match in magnitude would yield a match in phase as one is related to the other through

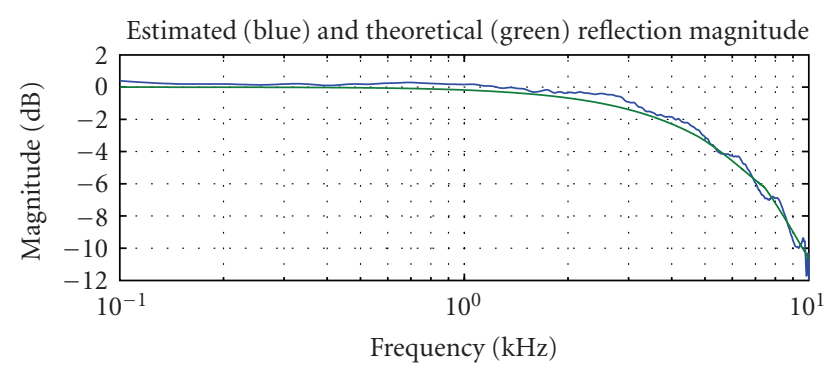

(a)

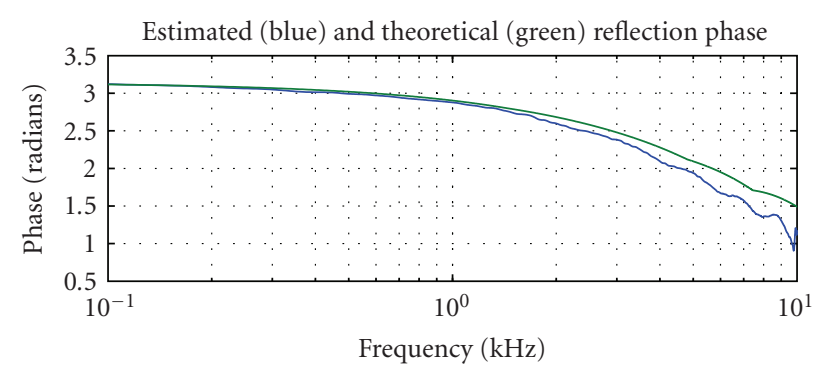

(b)

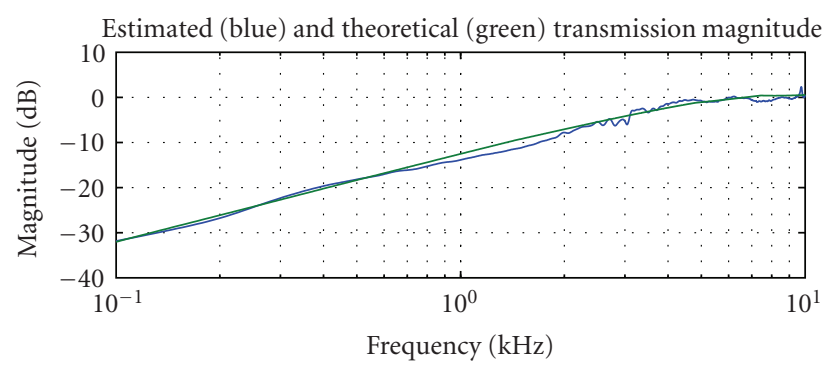

(c)

FIGURE 8: Estimated (blue) and theoretical (green, smooth curve) open-end cylinder reflection magnitude (a) and phase (b). Theory is based on the Levine and Schwinger approximation [33] with suitable expressions found in [12]. The estimated transmission magnitude (blue) is plotted with the amplitude complement (this assumption is valid for cylinders only) of the theoretical (green, smooth curve) reflection (c).

the Hilbert Transform. As the minimum-phase property is very desirable for synthesis, in part because it ensures invertibility, it is one that is imposed on any filters fit to the measurements made here.

\section{Appending the Trombone Bell}

A trombone bell is attached to the open end of the tube, with measurement yielding the arrival sequence shown in Figure 9. The arrivals follow the same pattern as those for the open tube, but with the bell reflection replacing the open cylindrical reflection, $R_{L}(\omega)=R_{b}(\omega)$.

The bell reflection function may be estimated by taking the spectral ratio of the second arrival $B_{0,2}(\omega)$ to that of the closed tube $C_{2}(\omega)$, as was done for the cylindrical open end 


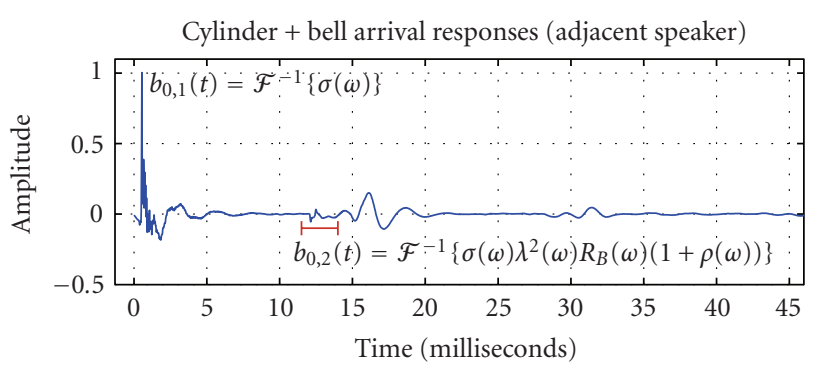

(a)

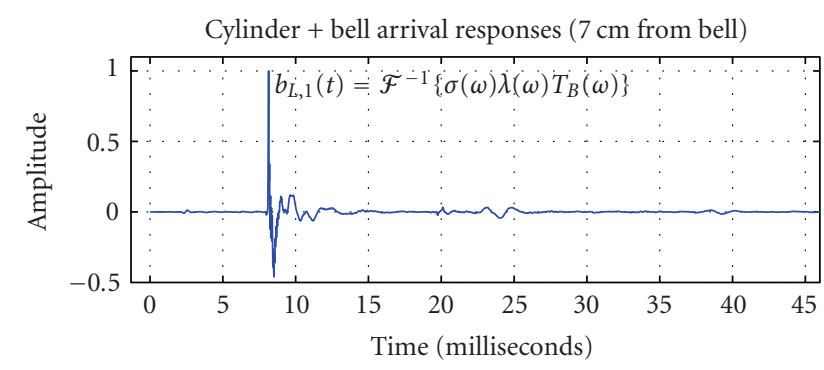

(b)

Figure 9: Arrival responses of the cylinder and bell at speaker (a) and outside the bell (b). Notice the low-amplitude effects of the junction reflection just before the higher-amplitude bell reflection in the second arrival ((a), underlined at approximately 12-14 milliseconds).

in Section 3.3. That is, the estimated bell reflection $\hat{R}_{b}(\omega)$ is theoretically given by

$$
\hat{R}_{b}(\omega)=\frac{B_{0,2}(\omega)}{C_{2}(\omega)}=\frac{\sigma(\omega) \lambda^{2}(\omega) R_{b}(\omega)(1+\rho(\omega))}{\sigma(\omega) \lambda^{2}(\omega)(1+\rho(\omega))} .
$$

This would be the case if a discontinuity were not created by appending the bell to cylinder. That is, though the radii of the cylinder and bell provide a good nested fit (there is no leak created at their junction) the bell radius at the bell's small end is not precisely flush with cylinder radius, with the difference being sufficient to create a reflection and transmission at their junction. The bell reflection estimated using the ratio of second arrivals in (14) would therefore also include the effects of this junction. This may be seen in the second arrival of the impulse response $b(t)_{0,2}$, which shows a downward followed by an upward pulse before the onset of the actual effects of the bell (see $b(t)_{0,2}$ of Figure 9(a)).

Though the effects of the junction likely have very little perceptible consequence, it is worthwhile to develop a method that accounts for the junction so that other acoustic objects/bells having a possibly greater mismatch to the tube's cross-sectional area may also be appended and more precisely estimated. The processing method described below is applied to measurements of another tube structure, constructed by appending a larger, but shorter, cylindrical tube section to the end to the two-meter tube (shown in Figure 10). As the junction between two cylinders and the open-end cylindrical reflection is well described theoretically, a comparison between theory and estimated results is again made to validate the processing technique.

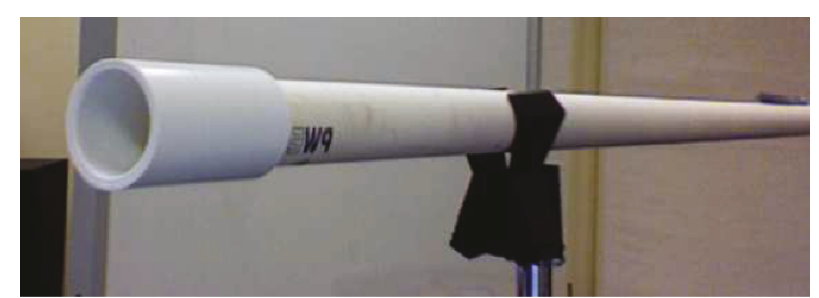

Figure 10: Instrument I3 is created to verify results that account for the junction created by appending a second tube object to the original measurement tube. It has a cylindrical open-end reflection which may, like that of instrument I2, be validated through comparison with theoretical expectation.

4.1. Estimating Open-End Reflectance in the Presence of a Junction Using Instrument I3. To isolate the open-end reflection $R_{L}(\omega)$ and transmission $T_{L}(\omega)$ in the presence of a junction, instrument transfer functions (1) and (2) are modified to account for the reflection and transmission occurring at the discontinuity between two adjacent cylindrical segments having cross-sectional area $S_{1}$ and $S_{2}$, respectively. As shown in Figure 11, a reflection and transmission will occur, with the reflection coefficient approximated by

$$
k=\frac{S_{1}-S_{2}}{S_{1}+S_{2}} .
$$

Assuming a sufficiently large $M$ to isolate the second arrival, that is, that once again the second arrival decays sufficiently before the onset of the third, the transfer function of the second arrival to the input $H_{k, 2}=Y_{0,2} / X$ at the instrument base, accounting for junction reflection coefficient $k$, may be expressed in the $z$-domain as

$$
\begin{aligned}
H_{k, 2}(z) & =\frac{Y_{0,2}(z)}{X(z)} \\
& =\left(1+R_{0}\right) \lambda_{M}^{2}\left[k+\frac{R_{L}\left(1-k^{2}\right) \lambda_{N}^{2} z^{-2 N}}{1+R_{L} k \lambda_{N}^{2} z^{-2 N}}\right],
\end{aligned}
$$

and an estimated open-end reflection function given by

$$
\hat{R}_{L, k}(z)=-\frac{\tilde{H}_{2}}{\left(\tilde{H}_{2} k-\left(1-k^{2}\right)\left(1+R_{0}\right) \lambda_{M}^{2}\right) \lambda_{N}^{2} z^{-2 N}},
$$

where

$$
\widetilde{H}_{2}=H_{k, 2}-\left(1+R_{0}\right) \lambda_{M}^{2} k
$$

and $R_{0}, R_{L}, \lambda_{M}$, and $\lambda_{N}$ are all functions of $z$ (omitted for brevity). Figure 12 shows how closely using the ratio of second arrivals (14) compares with theory and how accounting for the junction using (17) can improve the match.

The transmission function may be similarly estimated using the first arrival only, which arrives at the microphone (placed $7 \mathrm{~cm}$ outside the second tube) after a delay corresponding to the time taken to travel the length of the first and second tubes. The transfer function of the first arrival to the input $G_{k, 1}=Y_{L, 1} / X$ at the instrument output, accounting 


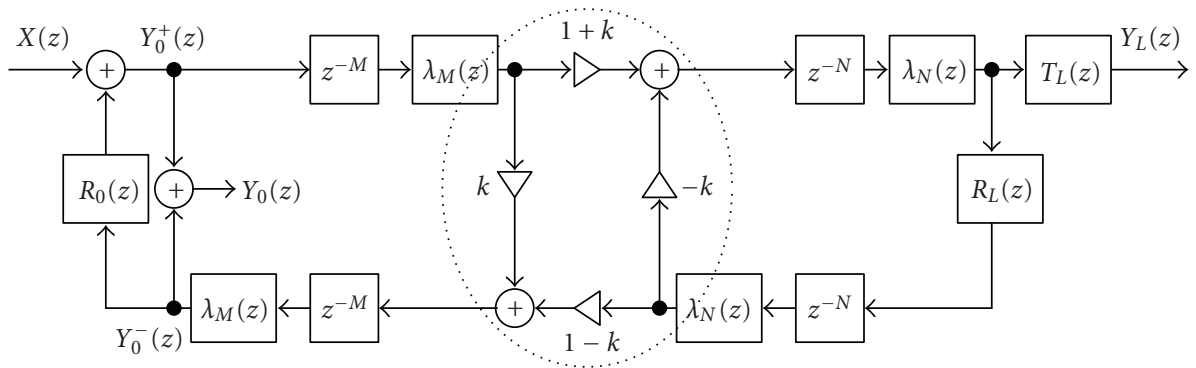

Figure 11: A signal flow diagram of two adjoined cylindrical tubes (instrument I3), with a two-port scattering junction due to the difference in the tube's cross-sectional area. With the junction removed (or equivalently by setting $k=0$ ), the diagram is equivalent to the instrument waveguide model in Figure 1.

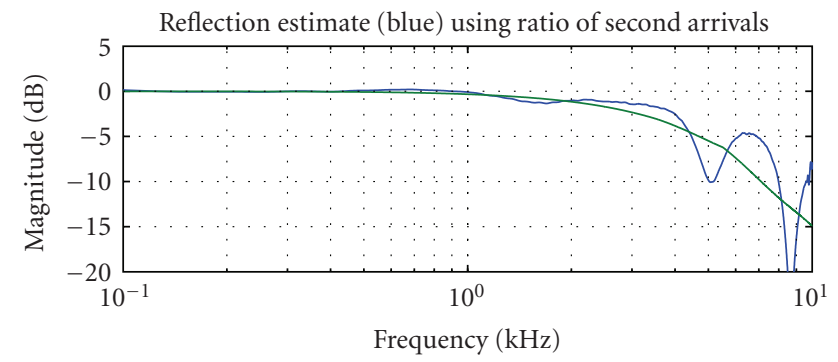

(a)

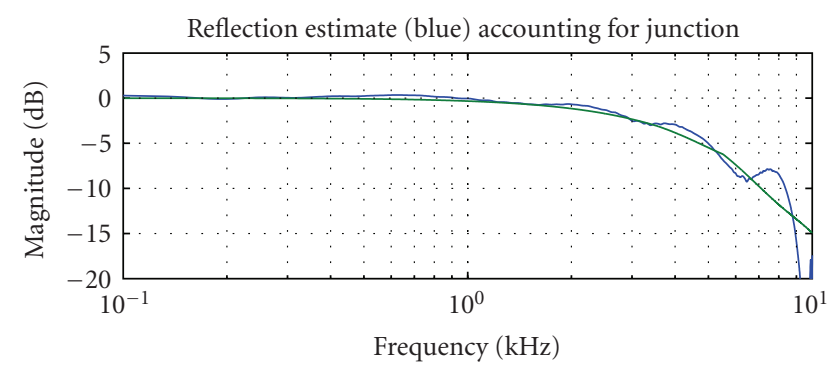

(b)

Figure 12: Estimated and theoretical open-end cylinder reflection magnitude as calculated using the ratio of second arrivals (a) according to (14) and by accounting for the discontinuity created by appending a larger radius tube (b) using (17). Notice the latter produces a better fit to the theoretical reflection.

for junction reflection coefficient $k$, is given in the $z$-domain by

$$
\begin{aligned}
G_{k, 1}(z) & =\frac{Y_{L, 1}(z)}{X(z)} \\
& =\frac{T_{L}(1+k) \lambda_{M}(z) \lambda_{N}(z) z^{-(M+N)}}{1+R_{L}(z) k \lambda_{N}^{2}(z) z^{-2 N}},
\end{aligned}
$$

yielding the estimated transmission transfer function

$$
\widehat{T}_{L, k}(z)=\frac{G_{k, 1}(z)+G_{k, 1}(z) R_{L}(z) k \lambda_{N}^{2}(z) z^{-2 N}}{(1+k) z^{-(M+N)}} .
$$

As shown in Figure 13, the effects of the junction are less significant when estimating the transmission function, with both methods (13) and (20) producing very similar results. This is not surprising since the recursive effects of

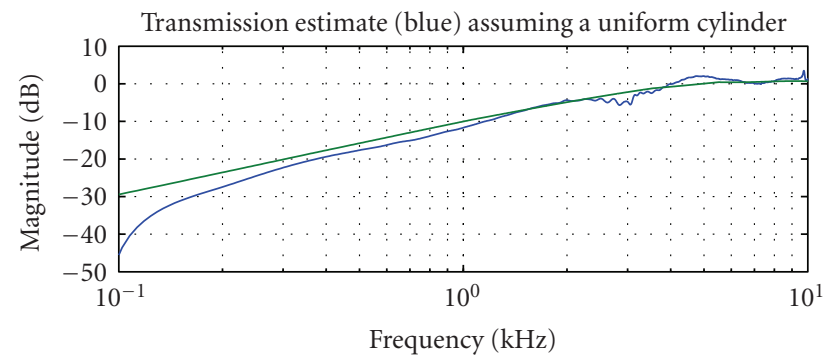

(a)

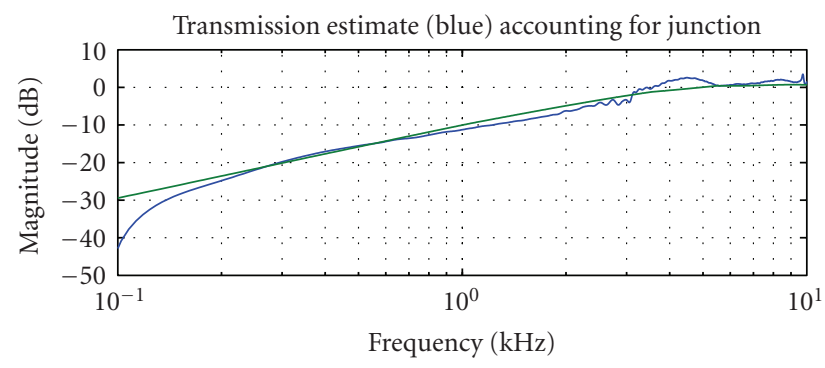

(b)

Figure 13: Estimated and theoretical open-end cylinder transmission magnitude as calculated using (13), (a), and by accounting for the junction created by appending a larger radius tube using (20), (b). Notice there is less of a discrepancy between the two methods than was seen for the reflection shown in Figure 12. A slight measurement error is observable at approximately $5 \mathrm{kHz}$, when the signal is higher than $0 \mathrm{~dB}$.

the junction (already minimal in this case) in the first arrival transmitted from the bell $Y_{L, 1}$ are limited to the pressure circulating in the second tube section, while the effects of the junction inside the tube at the mouthpiece $Y_{0,2}$ include pressure that has circulated in both tube sections, as well as the round-trip propagation from the speaker end to the open end and back.

It should be noted that complete models were also developed for transfer functions $H_{k}$ and $G_{k}$. It was found, however, that estimating $\widehat{R}_{L, k}$ and $\widehat{T}_{L, k}$ from $H_{k, 2}$ and $G_{k, 1}$, respectively, yielded slightly better results, likely because the first and second arrivals are not subject to the measurement noise that would result as the SNR deteriorates with each subsequent arrival in the entire impulse response. 


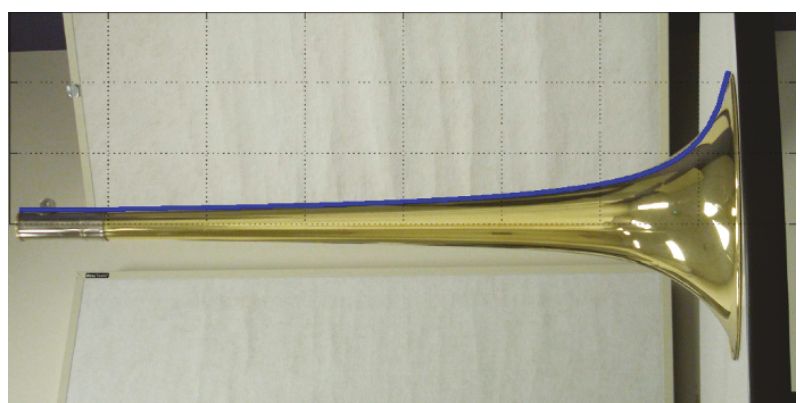

FIGURE 14: The profile of the bell shows a close fit to the equation for the Bessel horn, given in (21), using the parameters from Table 1.

4.2. Trombone Bell Model and Measurement. The close match achieved between theory and measurement for the adjoined cylinders described above provides confidence that the same measurement and processing technique may also be applied to acoustic elements having more complex shapes such as the trombone bell studied here. That is, the bell reflection and transmission can be obtained using (17) and (20).

Though it is more difficult to validate the bell measurement with theory, as the theory becomes increasingly approximate for shapes departing from the purely cylindrical or conical, it is nonetheless interesting and worthwhile to observe consistencies in behaviour between the measurement/estimation obtained here and approximate theoretical expectation. To provide this comparison, a one-dimensional model is developed from which bell reflection and transmission characteristics may be obtained. This is then compared with the estimated reflection and transmission obtained using (17) and (20).

For computational efficiency, musical horns are frequently modeled in one dimension, with models either based on Webster's equation $[16,34,35]$ or a piecewise connection of cylindrical or conical sections corresponding to the bell's profile $[15,23]$. Since both piecewise conical and horn function methods allow for the modeling of spherical wavefronts, they are expected to provide better accuracy, and as shown in [16], both methods produce very similar results. Here, the bell is modeled using a piecewise connection of conical segments.

As shown in Figure 14, the profile of the bell modeled here is well described by the so-called Bessel horn,

$$
a(x)=b\left(x+x_{0}\right)^{-\gamma},
$$

where $x_{0}$ is the position of the mouth of the horn, $x$ is the distance from the horn mouth, and $a(x)$ is the radius over the length of the bell. The variables $b$ and $x_{0}$ are chosen to give the correct radii at the small and large ends, while $\gamma$ defines the rate of flare [36]. Bessel horn parameters used here are provided in Table 1, with the resulting curve showing a good match to the trombone bell profile in Figure 14.

The bell is modeled using a piecewise connection of $N$ conical sections (or frustums) where, assuming both constant pressure and incoming volume velocities at the
Table 1: Parameters for Bessel horn described by (21) best fitting the trombone bell.

\begin{tabular}{lcc}
\hline Quantity & Variable & Value \\
\hline Length of the bell $(\mathrm{m})$ & & .502 \\
Radius at bell mouth $(\mathrm{m})$ & & .108 \\
Radius at small end $(\mathrm{m})$ & $\gamma$ & .01 \\
Bell flare constant & $x_{0}$ & .7 \\
Position of the bell mouth $(\mathrm{m})$ & $b$ & .0174 \\
Fitting parameter & $b .0063$ \\
\hline
\end{tabular}

junctions, the relationship among traveling waves in adjacent sections may be written in matrix form as

$$
\left[\begin{array}{c}
p_{n}^{+} \\
p_{n}^{-}
\end{array}\right]=\mathbf{A}_{n}\left[\begin{array}{c}
p_{n+1}^{+} \\
p_{n+1}^{-}
\end{array}\right],
$$

where the scattering matrix is given by

$$
\mathbf{A}_{n}=\left[\begin{array}{ll}
\frac{Z_{n}}{Z_{n+1}} \frac{Z_{n+1}+Z_{n}^{*}}{Z_{n}+Z_{n}^{*}} e^{j k L_{n+1}} & \frac{Z_{n}}{Z_{n+1}^{*}} \frac{Z_{n+1}^{*}-Z_{n}^{*}}{Z_{n}+Z_{n}^{*}} e^{-j k L_{n+1}} \\
\frac{Z_{n}^{*}}{Z_{n+1}} \frac{Z_{n+1}-Z_{n}}{Z_{n}+Z_{n}^{*}} e^{j k L_{n+1}} & \frac{Z_{n}^{*}}{Z_{n+1}^{*}} \frac{Z_{n+1}^{*}+Z_{n}}{Z_{n}+Z_{n}^{*}} e^{-j k L_{n+1}}
\end{array}\right],
$$

for a section of length $L_{n}$ and with complex wave impedance $Z_{n}$. For a model having $N$ sections, $N-1$ scattering matrices are multiplied,

$$
\left[\begin{array}{c}
p_{1}^{+} \\
p_{1}^{-}
\end{array}\right]=\mathbf{A}_{1} \mathbf{A}_{2} \cdots \mathbf{A}_{N-1}\left[\begin{array}{c}
p_{N}^{+} \\
p_{N}^{-}
\end{array}\right],
$$

to yield the model's single final scattering matrix

$$
\mathbf{P}=\prod_{n=1}^{N-1} \mathbf{A}_{n}
$$

relating the bell input and output traveling pressure waves.

The expression for the reflection function of the bell may be formed from the above model by taking the ratio of the wave reflected by the bell $p_{1}^{-}$to the bell input wave $p_{1}^{+}$,

$$
\begin{aligned}
R_{B}=\frac{p_{1}^{-}}{p_{1}^{+}} & =\lambda^{2}(\omega) \frac{p_{N}^{+} \mathbf{P}_{2,1}+p_{N}^{-} \mathbf{P}_{2,2}}{p_{N}^{+} \mathbf{P}_{1,1}+p_{N}^{-} \mathbf{P}_{1,2}} \\
& =\lambda^{2}(\omega) \frac{\mathbf{P}_{2,1}+\mathbf{P}_{2,2} R_{L}(\omega)}{\mathbf{P}_{1,1}+\mathbf{P}_{1,2} R_{L}(\omega)},
\end{aligned}
$$

where the final expression is obtained by incorporating an open-end reflection at the termination of the $N$ th section by substituting $p_{N}^{-}=p_{N}^{+} R_{L}(\omega)$ and by commuting round-trip propagation losses $\lambda^{2}(\omega)$.

Similarly, the bell transmission is given by the ratio of the wave radiated out the bell $p_{N}^{+} T_{L}(\omega)$, where $\left.T_{L} \omega\right)$ is the openend transmission function, to the bell's input $p_{1}^{+}$,

$$
T_{B}(\omega)=\frac{p_{N}^{+} \lambda(\omega) T_{L}(\omega)}{p_{1}^{+}}=\frac{\lambda(\omega) T_{L}(\omega)}{\mathbf{P}_{1,1}+\mathbf{P}_{1,2} R_{L}(\omega)} .
$$




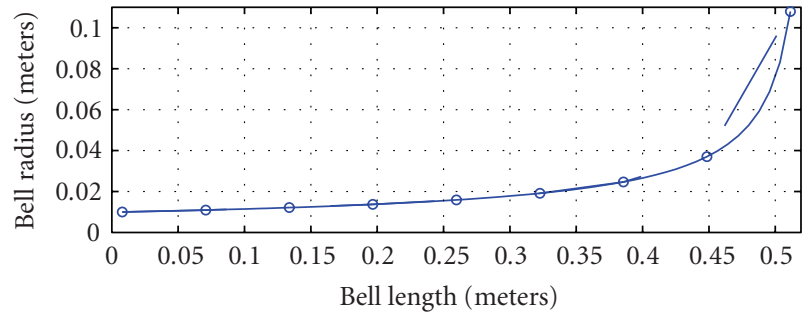

FIGURE 15: Geometry of bell (solid line) showing radii of eight piecewise conical/frustum segments and resulting modeled profile (dashed line).

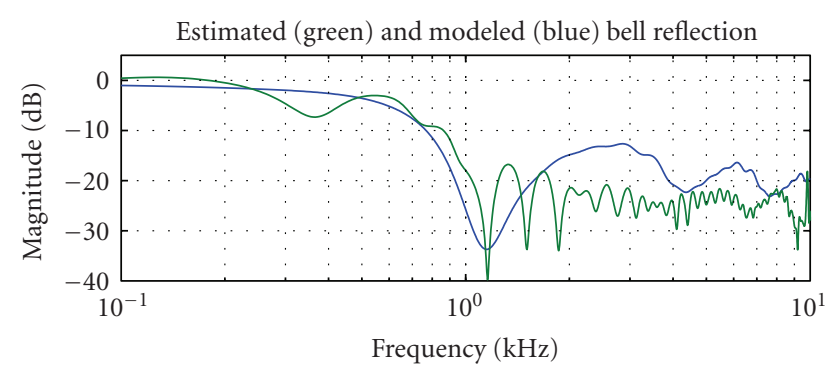

(a)

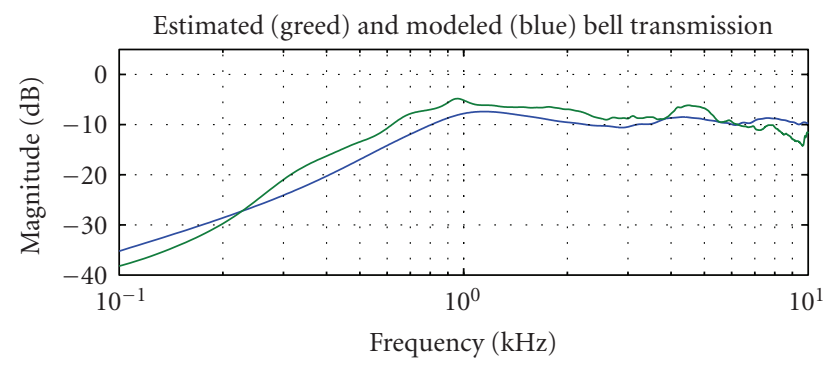

(b)

Figure 16: Estimated and modeled bell reflection (a) and transmission (b) magnitudes.

As in Section 3.3, suitable expressions for $R_{L}(\omega)$ and $T_{L}(\omega)$ may be found in [12].

The trombone bell is modeled using eight conical sections, with geometry and dimensions determined from the Bessel horn function (21) and Table 1, with profile plotted in Figure 15. The commuted propagation losses are modeled as described in [21], using the median of the bell profile radii and the bell length (from Table 2) as input parameters.

As mentioned above, it is not expected that the estimated and modeled bell reflection and transmission will match to the same degree as with the open cylinder, largely because the 1-D model does not take into account higher-order and/or evanescent modes. Indeed, if the match were too good, it would negate the need to measure the bell reflection and transmission for improved accuracy. Nevertheless, as shown in Figure 16, the magnitude of the modeled bell reflection (26) and transmission (27) functions displays very similar behaviour to that estimated from measurement, (17) and (20), respectively, providing confidence in the accuracy of the measurement and estimation technique.
TABLE 2: Trombone tubular sections (numbers correspond to parts in Figures 17 and 18) and dimensions, including top (t.) and bottom (b.) inner and outer slides, retracted and extended (ext.).

\begin{tabular}{lcc}
\hline Part & Length $(\mathrm{cm})$ & Radius $(\mathrm{cm})$ \\
\hline t. inner slide (1) & 70.8 & 0.69 \\
t. outer slide, ext. (2) & 53 & 0.72 \\
slide crook (3) & 17.7 & 0.74 \\
b. outer slide, ext. (4) & 53 & 0.72 \\
b. inner slide (5) & 71.1 & 0.69 \\
Gooseneck (6) & 24.1 & 0.71 \\
Tuning slide (7) & 25.4 & $0.75,1.07$ \\
Bell flare (8) & 56.7 & $1,10.8$ \\
\hline
\end{tabular}

\section{Trombone Model and Measurement}

With the trombone bell measurement providing both reflection and transmission transfer functions and theoretical propagation losses for cylinders with a different cross section, it is possible to assemble results to complete the instrument transfer functions described in (1) and (2) for the trombone. The only value yet unknown is the reflection at the mouthpiece $R_{0}(\omega)$. As this is expected to change during performance with the vibrating lips changing both the mouthpiece volume and the opening to the bore, it is not suitably obtained using the methods described here, but rather is left for work currently in progress, whereby the generalized valve model [9] is configured and coupled to the trombone instrument model presented here.

A complete trombone (mouthpiece omitted) is shown in Figure 18, with corresponding trombone components and dimensions provided in Table 2. Figure 17 shows an interior view of the complete trombone in both retracted and extended positions, producing bores with effective lengths of $209.1 \mathrm{~cm}$ and $315.1 \mathrm{~cm}$, respectively, with asterisks showing possible cylindrical junctions that may or may not be considered depending on the desired level of accuracy. Trombone components 1-7 in Figure 17 are modeled as cylindrical waveguide sections, following dimensions in Table 2 for appropriate delay length and radius, parameters used for the propagation loss model described in [21].

In future work, it would be very interesting to compare the results of the trombone model presented here, in retracted, extended, and intermediate positions, with an accurately obtained input impedance of the complete trombone. In the meantime, however, the validation of the model and measurement of the trombone's composite elements provides confidence that their assembly into the real-time model, described by (1) and (2), contributes an accurate, real-time interactive virtual instrument suitable for control parameter estimation and, when combined with a lipvalve model, interactive sound production, both significant applications in real-time music performance.

\section{Conclusions}

The trombone model presented here consists of two trombone instrument transfer functions, one taken at the position 


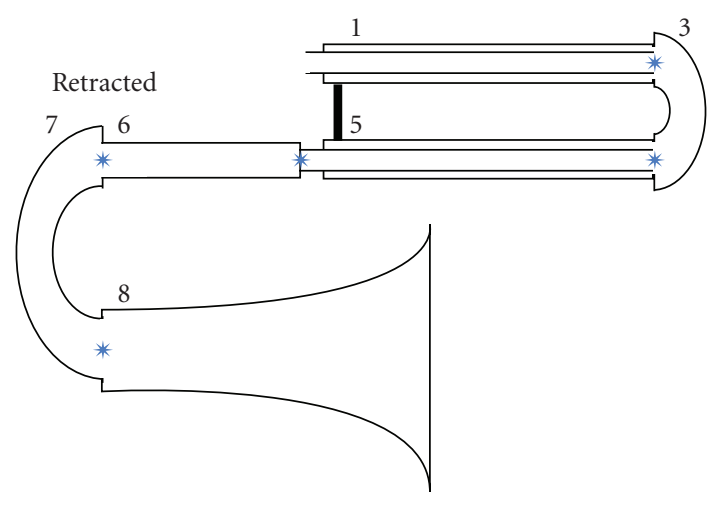

(a)

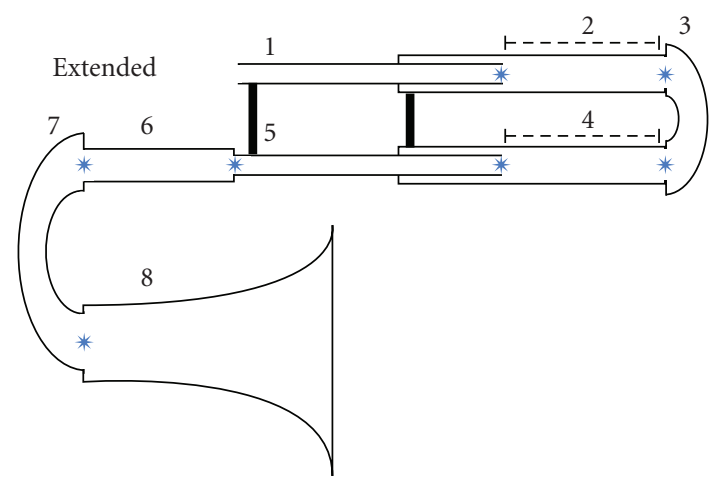

(b)

FIGURE 17: Interior view of trombone, in both fully retracted and fully extended positions, showing assembly of components from Table 2.

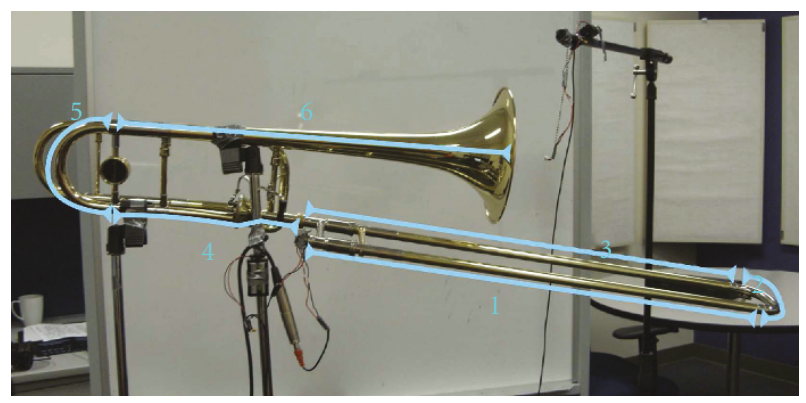

Figure 18: Trombone tubular components corresponding to Table 2.

of the mouthpiece for dynamic coupling to a lip-valve model and one taken outside the bell for the production of the transmitted sound and for inverse modeling applications. Model transfer functions consist of several dynamic and static unknown acoustic waveguide elements due to frequency-dependent propagation and boundary conditions. Those expected to change during performance, such as the effective bore length with the moving hand slide, are made suitably parametric, with theoretical propagation losses for cylinders being dependent on both tube length and radius. Elements not expected to change during performance, such as bell reflection and transmission, are obtained by extending a previously introduced measurement technique shown to be accurate for cylindrical and conical sections, but further developed here for the estimation of more complex acoustic tube structures.

The model elements estimated from measurement are validated by comparison with theoretical expectation. For the cylindrical case, which is well described theoretically, the propagation losses and open-end cylindrical reflection/transmission are shown to produce a very close match to theory. This both validates the measurement/estimation technique and provides confidence that theoretically modeled propagation losses may be used for cylindrical/conical sections in lieu of measured data (should measurements be difficult to obtain), with little consequence to synthesis accuracy. For efficiency and interactivity in real-time performance, in fact, it is likely preferable to use a theoretically based parametric filter for propagation losses such as that described in [21].

In the case of the trombone bell, the flared opening departs considerably from the purely cylindrical or conical sections of the bore and has no one-parameter traveling wave solution. The acoustic behaviour of such structures cannot be completely described using a one-dimensional model, as such a model would not capture higher-order and/or evanescent modes. Nevertheless, bell reflection and transmission functions are estimated from measurement and then compared with a one-dimensional bell model developed using a piecewise connection of conical segments corresponding to the bell profile. The bell model is expressed in the frequency domain, using a matrix notation from which expressions for bell reflection and transmission transfer functions are obtained algebraically. Though it is not expected that the bell model and measurement comparison will yield the close match seen for the unflanged open-end boundary condition at the end of a cylindrical pipe, the comparison does show consistent behaviour. Considering the prior validation of the measurement technique for cylindrical structures better described theoretically, the response of the bell based on measurement is expected to be more accurate than that described by the one-dimensional model.

Though it is the magnitude of the frequency response that is presented here, this in no way implies that the phase is not important. Rather, it has been found that filter elements possess the minimum-phase property, or at least approximately so, making plots of their phase redundant to the magnitude, as they are related by a shift of 90 degrees. A match in magnitude, therefore, implies a match in phase as well. This desirable property also assures that, by definition, the elements are invertible and well posed in terms of causality and stability.

In this work, a parametric synthesis model of the trombone is developed, focusing both on accuracy and efficiency, making it suitable for real-time computer music applications. In particular, it is currently being used to determine suitable input parameters for the generalized pressure-controlled valve model, described in [9], with the trombone model here providing an instrument body for the "blown open" configuration of the valve. It is also being used in developing inverse modeling strategies to extract 
a lip-valve signal-a signal corresponding to the pressure input into the bore at the mouthpiece-using only the signal recorded from the trombone during real-time performance. This will allow trombone performers to generate computer input parameters (perhaps to another synthesis model) by making subtle changes to embouchure and input pressure, allowing them either to extend their own instrument through effects processing or perhaps to control another virtual instrument altogether. The trombone is a particularly wellsuited instrument for this goal because, unlike other wind instruments having toneholes and a myriad of possible fingerings, the trombone is well described by a relatively simple waveguide with only a length that changes with a movement of the slide during performance. Trombone synthesis by model and measurement provides a parametric model that is accurate, efficient, and interactive, making it suitable for both interactive sound production and inversemodeling (parameter estimation), both having considerable significance to applications in real-time music performance.

\section{Acknowledgments}

The authors would like to sincerely thank the anonymous reviewers of this article, whose diligence and attention to detail have greatly strengthened the presentation of this work. Jonathan Abel continues to impact this research through ever-illuminating discussion and insight. In addition, the authors would like to acknowledge the support of the Natural Sciences and Engineering Research Council of Canada (NSERC).

\section{References}

[1] M. Karjalainen, V. Välimäki, B. Hernoux, and J. Huopaniemi, "Explorations of wind instruments using digital signal processing and physical modeling techniques," Journal of New Music Research, vol. 24, no. 4, pp. 301-317, 1995.

[2] T. Helie, C. Vergez, J. Levine, and X. Rodet, "Inversion of a physical model of a trumpet," in The 38th IEEE Conference on Decision and Control (CDC), pp. 2593-2598, December 1999.

[3] S. Serafin, J. O. Smith, and H. Thornburg, "A pattern recognition approach to invert a bowed string physical model," in Proceedings of the International Symposium on Musical Acoustics (ISMA '01), Perugia, Italy, September 2001.

[4] S. Serafin and D. Young, "Bowed string physical model validation through use of a bow controller and examination of bow strokes," in Proceedings of the Stockholm Music Acoustics Conference (SMAC '03), Sweden: Stockholm, August 2003.

[5] G. Scavone and A. da Silva, "Frequency content of breath pressure and implications for use in control," in Proceedings of the International Conference on New Interfaces for Musical Expression (NIME '05), pp. 93-96, Vancouver, Canada, May 2005.

[6] E. Guaus, J. Bonada, A. Perez, E. Maestre, and M. Blaauw, "Measuring the bow pressing force in areal violin performance," in Proceedings of the International Symposium on Musical Acoustics (ISMA '07), Barcelona, Spain, September 2007.

[7] M. van Walstijn and G. de Sanctis, "Towards physicsbased re-synthesis of woodwind tones," in Proceedings of the 19th
International Congress on Acoustics, Madrid, Spain, September 2007.

[8] T. Smyth and J. Abel, "Estimating the reed pulse from clarinet recordings," in Proceedings of th International Computer Music Conference (ICMC '09), pp. 235-238, Montreal, Canada, August 2009.

[9] T. Smyth, J. Abel, and J. O. Smith, "A generalized parametricreed model for virtual musical instruments," in Proceedings of th International Computer Music Conference (ICMC'05), pp. 347-350, Barcelona, Spain, September 2005.

[10] W. G. Gardner, "Efficient convolution without input-output delay," Journal of the Audio Engineering Society, vol. 43, no. 3, pp. 127-136, 1995.

[11] T. Smyth and J. Abel, "Convolutional synthesis of wind instruments," in Proceedings of the IEEE Workshop on Applications of Signal Processing to Audio and Acoustics (WASPAA '07), New Paltz ,NY, USA, October 2007.

[12] T. Smyth and J. S. Abel, "Estimating waveguide model elements from acoustic tube measurements," Acta Acustica United with Acustica, vol. 95, no. 6, pp. 1093-1103, 2009.

[13] J. O. Smith, "Physical audio signal processingfor virtual musical instruments and audio effects," December 2008, http://ccrma.stanford.edu/ jos/pasp/.

[14] V. Välimäki, J. Pakarinen, C. Erkut, and M. Karjalainen, "Discrete-time modelling of musical instruments," Reports on Progress in Physics, vol. 69, no. 1, pp. 1-78, 2006.

[15] G. R. Putland, "Every one-parameter acoustic field obeys Webster's horn equation," Journal of the Audio Engineering Society, vol. 41, no. 6, pp. 435-451, 1993.

[16] D. Berners and J. O. Smith, "On the use of Schrödinger's equation in the analytic determination of horn reflectance," in Proceedings of the International Computer Music Conference (ICMC'94), pp. 419-422, Aarhus, Denmark, September 1994.

[17] T. Hélie, "Unidimensional models of acoustic propagation in axisymmetric waveguides," Journal of the Acoustical Society of America, vol. 114, no. 5, pp. 2633-2647, 2003.

[18] V. Välimäki, Discrete-time modeling of acoustictubes using fractional delay filters, Ph.D. dissertation, Helsinki University of Technology, Faculty of Electrical Engineering, Laboratory of Acoustic and Audio Signal Processing, Espoo, Finland, 1995, report no. 37.

[19] G. P. Scavone, An acoustic analysis of single-reed woodwind instruments with an emphasis on designand performance issues and digital waveguide modeling techniques, Ph.D. dissertation, Music Department, Stanford University, Stanford, Calif, USA, 1997, available as CCRMA Technical Report no. STAN-M100.

[20] R. Mignot, T. Hélie, and D. Matignon, "Digital wave guide modeling for wind instruments: building a state space representation based on the Webster-Lokshin model," IEEE Transactions on Audio, Speech and Language Processing, vol. 18, no. 4, pp. 843-854, 2010.

[21] J. Abel, T. Smyth, and J. O. Smith, "A simple, accurate wall loss filter for acoustic tubes," in Proceedings of the International Conference on Digital Audio Effects (DAFX '03), pp. 53-57, London, UK, September 2003.

[22] R. Causse, J. Kergomard, and X. Lurton, "Input impedance of brass musical instruments—comparison between experiment and numerical models," The Journal of the Acoustical Society of America, vol. 75, no. 1, pp. 241-254, 1984.

[23] D. P. Berners, Acoustics and signal processing techniquesfor physical modeling of brass instruments, Ph.D. dissertation, Stanford University, Stanford, Calif, USA, 1999. 
[24] D. B. Sharp, Acoustic pulse reflectometry for the measurement of musical wind instruments, Ph.D. dissertation, University of Edinburgh, 1996.

[25] J.-P. Dalmont, "A new impedance sensor for wind instruments," Journal of the Acoustical Society of America, vol. 123, no. 5, p. 3014, 2008.

[26] T. Ossman, H. Pichler, and G. Widholm, "Bias: a computeraided test system for brass wind instruments," Audio Engineering Society Convention 87, paper no. 2834, October 1989.

[27] T. Smyth and J. Abel, "Observing the effects of waveguide model elements in acoustic tube measurements," in Proceedings of the 152nd Meeting of the Acoustical Society of Americal ASA '06), vol. 120, p. 3331, Honolulu, Hawaii, November 2006.

[28] T. Smyth and J. Abel, "Extending the generalized reed model with measured reflection functions," in Proceedings of th International Computer Music Conference (ICMC '07), pp. 252-255, Copenhagen, Denmark, August 2007.

[29] X. Rodet and C. Vergez, "Physical models of trumpetlike instruments: detailed behavior and model improvements," in Proceedings of the International Computer Music Conference (ICMC '96), Clear Water Bay, Hong-Kong, August 1996.

[30] C. Vergez and X. Rodet, "Comparison of real trumpet playing, latex model of lips and computer model," in Proceedings of th International Computer Music Conference (ICMC '97), Thessaloniki, Greece, September 2007.

[31] A. Farina, "Simultaneous measurement of impulse response and distortion with a swept-sine technique," in Proceedings of the 108th AES Convention, pp. 18-22, Paris, France, February 2000.

[32] A. H. Benade, "On the propagation of sound waves ina cylindrical conduit," Journal of the Acoustical Societyof America, vol. 44, no. 2, pp. 616-623, 1968.

[33] H. Levine and J. Schwinger, "On the radiation of sound from an unflanged circular pipe," Physical Review, vol. 73, no. 4, pp. 383-406, 1948.

[34] A. H. Benade and E. V. Jansson, "On plane and spherical waves in horns with nonuniform flare i. theory of radiation, resonance frequencies, and mode conversion," Acustica, vol. 31, no. 2, pp. 80-98, 1974.

[35] M. van Walstijn and V. Välimäki, "Digital waveguide modeling of flared acoustical tubes," in Proceedings of th International Computer Music Conference (ICMC '97), pp. 196-199, Thessaloniki, Greece, September 2007.

[36] N. H. Fletcher and T. D. Rossing, The Physics of Musical Instruments, Springer, New York, NY, USA, 1995. 